\title{
Complex Adaptive Matter: Emergent Phenomena in Materials
}

\section{Daniel L. Cox and David Pines, Guest Editors}

\begin{abstract}
In the study of matter, both living and inanimate, the breakthrough discoveries and most scientists' intellectual obsessions often flow from what we call emergent behavior phenomena not readily predictable from a detailed knowledge of the material subunits alone. We call systems that display emergent behavior complex adaptive matter, and their relevant organizing principles are unique to their scales of length and time. This issue of MRS Bulletin provides an overview of the aggregate of research on complex adaptive matter through a survey of five examples, ranging from intrinsically disordered electron matter in high-temperature superconductors to protein aggregates in amyloid diseases like Alzheimer's. We explain the philosophy and motivation for this research, noting that the study of emergent phenomena complements a globally reductionist scientific approach by seeking to identify, with intellectual precision, the relevant organizing principles governing the behavior. Our authors focus on the character of emergence for their particular systems, the role of materials research approaches to the problems, and the efforts to identify the organizing principles at work.
\end{abstract}

Keywords: complex adaptive matter, emergent behavior.

\section{Introduction}

In the study of matter, both living and inanimate, the breakthrough discoveries and most scientists' intellectual obsessions often flow from what we call emergent behavior: phenomena that owe their existence to interactions between many subunits, but whose existence cannot be deduced from a detailed knowledge of those subunits alone. A classic scientific question that illustrates this notion of emergence is how can an assembly of molecules (us) actually know just what a molecule is? Surely, consciousness and cognition are emergent phenomena. As another example, consider high-temperature superconductivity, a phenomenon that has been known for nearly two decades, but that still eludes a comprehensive understanding. No quantum mechanical calculations predicted the existence of this remarkable phase of matter, and to date, no microscopic calculations or numerical simula- tions have conclusively post-dicted it, either, despite the efforts of many brilliant minds working on the problem.

We call materials exhibiting emergent behavior complex adaptive matter, and their relevant organizing principles are characterized by given scales of length and time. This issue of MRS Bulletin is organized around the research problems in this area and the general theme of emergent phenomena in matter, rather than a specific class of material or materials-related problems.

The ordering of the articles in this issue roughly follows a progression of length scales, from atomic-scale modulation of electron density in the cuprate superconductors to the large-scale protein aggregates visible in the brains of victims of amyloid diseases such as Alzheimer's. In each case, our authors have focused on the character of emergence for their particular systems, the role of materials research approaches to the problems, and the efforts to identify the organizing principles governing these phenomena.

Before we turn to overviews of the individual articles, we should explain our choice of terms as well as the philosophy behind the study of complex adaptive matter. We ask the indulgence of the reader for this philosophical excursion.

\section{Complex Adaptive Matter and Emergence:Terms and Philosophy}

In a very real sense, emergence represents a democracy of scale: no length scale, time scale, or particle is more fundamental than any other. ${ }^{1,2,3}$ For example, the study of the properties of electrons in isolation is a wonderful research area, but one that has not turned out to be a sufficient guide to understanding the electronic properties of the cuprate superconductors or the socalled heavy fermion materials that are discussed in this issue. The reason is that collections of atoms and electrons generate new states classifiable by momentum, energy, and other familiar quantities that sometimes have little obvious relation to the individual particles. Hence, a full intellectual appreciation of emergence requires a complement to the reductionist dream of breaking matter into its most fundamental pieces and reassembling them to gain understanding. ${ }^{4}$ Scientific reductionism as an approach to understanding matter has led to remarkable perspectives on the universe and the smallest particles and has given us the novel realms of relativistic phenomena and the quantum. Still, we must work with materials on a daily basis whose properties are characterized by new organizing principles that give rise to quite unexpected emergent behavior that is not manifestly connected to, say, quarks or superstrings. ${ }^{5}$

To illustrate what we mean by this, we note, for example, that there is no automatic way to design novel electronic materials by merely coding the details of the atoms into a computer and solving the Schrödinger equation; rather, the skilled electronic-structure theorist must think deeply for each new system and use the Schrödinger equation to that end. Similarly, we have been unable to design a functional protein molecule merely by trying out amino acid sequences and integrating equations of motion in time for the atoms according to Newton's laws and atomic force models. Even some of the most successful practitioners of scientific reductionism acknowledge that it doesn't function fully on its home turf: many particle physicists adopt the central goal of 
describing phenomena at particular length and time scales by categorizing them under various "effective field theories."

Accepting emergence, then, requires scientists who work on the fundamental properties of matter to humbly re-engage the oldest traditions of science. We must emphatically state that a focus on complex adaptive matter should not be construed as a rejection of reductionism per se, but rather as a response to the limitations of a global reductionist approach. By global reductionism, we mean the historic drive to continue to break matter into smaller and smaller pieces with the assumption that knowledge of the pieces can be reassembled into an understanding of the whole system at larger length scales. Indeed, we must acknowledge the primacy of experiment in producing new knowledge and direct our hypothesis-forming and theoretical energies toward local reductionism: identifying the key organizing principles of the given complex adaptive material before us, at its relevant length and time scales, armed with lessons derived from centuries of success of a global reductionist approach.

Global reductionism provides, for example, some organizing principles that transcend length scales, such as conservation laws. Because there are conservation laws for charge that permeate all length scales, it is helpful to know that quarks carry multiples of one-third the electron charge to assemble a predictive model of hadrons such as the proton. Similarly, because electrons carry quantized charge and magnetic moments (spin), we can use this fact to confidently characterize the composite "particles" called Cooper pairs of electrons that condense in the superconducting state or the particle-hole excitons in semiconducting materials in terms of their spin and charge states.

Of course, the development of quantitative descriptions of particular kinds of materials (as opposed to the global reductionist goal of all of matter) is a longstanding tradition for materials scientists and engineers. We would hope that our work builds on this tradition by fulfilling the criterion of success applied to the most celebrated reductionist theories, such as Darwinian evolution and Newtonian mechanics: they are themselves emergent marvels in giving back far more numerous predictions of phenomena than the observations of the actual phenomena that led to their formulation.

It is fair to ask whether emergence is inadvertent, in that human frailty prevents us from predicting some phenomenon beforehand that is, with some hindsight, knowable in principle. The other more dramatic possibility is that emergence is intrinsic: no current or future methodology could enable us to make the leap from well-understood phenomena at one length scale to imperfectly understood phenomena at another length scale, meaning that new concepts and methods are the only route to developing understanding. The honest answer is this: it doesn't matter! Our duty as researchers on complex adaptive matter is to seek discovery and new organizing principles even if we discover after the fact that some existing theory might have predicted them.

As an example of the limits of global reductionism, consider the $a b$ initio prediction of protein structure. If we first seek to solve the non-relativistic Schrödinger equation relevant at the atomic scale exactly for all the particles in the 42-aminoacid peptide implicated in the amyloid plaques of Alzheimer's disease, for example, we can forget about it without radical breakthroughs in computation. In the space of many-body wave functions, the basis set size (number of relevant coordinate axes) explodes at a faster than exponential rate with molecular size, dictating an unattainable time scale for solution. With approximate methods, such as density functional theory, overlaid with further approximations, we can achieve "order $N^{\text {" scaling }}{ }^{7}$ for the solution time for the ground-state energy, where $N$ is the number of electrons. However, it remains difficult to sample excited-state energetics with such schemes, which limits a full study of protein folding. Hence, there is little doubt that without a breakthrough in, say, quantum computing, existing paradigms render an $a b$ initio prediction of folded protein structures unobtainable, placing protein folding, in that sense, in the category of intrinsic emergence. At the same time, employing electronic-structure programs to thoughtfully study the properties of subunits of proteins can be a very useful exercise.

If we content ourselves with treating the peptide classically, writing down approximate force laws for the atoms, and integrating their motion subject to Newton's laws (using molecular dynamics programs, such as $\mathrm{CHARMM}^{8}$ or $\mathrm{AMBER}^{9}$ ), we find that cleverness can currently carry computer simulations into the time realm of hundreds of nanoseconds for large peptides $^{10}$ and hundreds of microseconds for small ones, ${ }^{11}$ sufficient to find final folded structures in some cases. However, there is considerable evidence that the reconfiguration time of large proteins and peptides involved in amyloid diseases can be on the scale of seconds, many orders of magnitude outside the current limits of computation for all-atom simulations. Moreover, the alert reader will already have noted that all-atom molecular dynamics in this form already implicitly abandons the dream of global reductionism, since the force fields that are used are by necessity semi-empirical, often biased toward particular structures that we know well and that are based upon ambient physiological conditions. This discussion of the fully classical approach to protein folding also supports the view of protein folding as an example of intrinsic emergence.

Protein folding also allows us to explain the depth of meaning and precision in the words "complex adaptive matter." There is no doubt that proteins are complex systems, compared with the most studied forms of inanimate matter such as monatomic elemental solids. The chemical diversity of the genetically coded amino acids, the ability to put them together in long heterogeneous sequences, and the receptiveness of many proteins to "posttranslational modification" after synthesis by attachment of non-amino-acid-based molecules (e.g., sugars or transition-metal complexes) certainly must be called "complex" by any observer. The great ingenuity of materials synthesists have given us nearrivals in complexity such as large-unit-cell superconductors, colossal magnetoresistance materials, zero-thermal-expansion materials, and multiferroics (materials with, e.g., ferroelectric and ferromagnetic order).

By "adaptive" here, we mean that these complex materials may adopt a variety of phases and yet robustly resist external perturbations within a given phase. In this sense, adaptive matter behaves like a living organism, with identifiable, unique properties and operational states that are robust against all but the most severe external perturbations. Within the language of the renormalization group theory of critical phenomena, the phases are said to be governed by "fixed-point Hamiltonians"12 that are energy functionals of the system, unchanged under a suitably defined rescaling of the volume. The significance of this rather esoteric language is that the properties of a system right at a critical point are unchanged at different length scales, as in the fluctuation spectrum of a liquid-gas mixture displaying critical opalescence. The renormalization group theory shows that these special critical points can govern the behavior of a system over a wide range of materials and thermodynamic parameters. In recent years, a broader terminology has been developed to characterize systems that may be in or out of equilibrium and are not 
macroscopically large. When they exhibit spatio-temporal phenomena that enjoy similar "protection" against perturbations, they are called protectorates. ${ }^{2,3}$

Turning back to protein matter for a moment, the fact that many proteins with very different sequences have the same function suggests that well-folded biological macromolecules may be classifiable by this notion of protectorates, a point that is worthy of further study. New protectorates seem to emerge on the nanoscale in inanimate materials as well; there are the famous examples of highly catalytic gold nanoparticles ${ }^{13}$ and ferroelectric niobium nanoparticles, ${ }^{14}$ as well as manifold examples of structures stabilized on the nanoscale that cannot be observed in bulk..$^{15}$

A beautiful aspect of the notion of the protectorate is that multiple-dimensional parameter spaces describing the energy of the system essentially collapse to lowdimensional spaces, so that if you find one mathematical model that happens to fall in the range of validity of a protectorate, you have found a good enough description of the phenomena. That is, while it may be true that, say, three-atom, fouratom, or five-atom interactions are in a literal sense present for a solid, those interactions can be mathematically irrelevant for a description of a phase of interest in the material. For example, the Bardeen-Cooper-Schrieffer (BCS) theory of ordinary superconductors needs but three parameters to describe the superconducting state: a set of electron-like, quantum "quasi-particle" states near the highest filled state of the metal; an energy gap for the excitation of quasi-particles out of the superconducting state; and a parameterization of the net attractive interaction between these quasi-particles, which induces them to "pair."16 Many magnets can be characterized almost completely by a handful of coupling parameters between localized magnetic moments. In these examples, the language of the renormalization group renders the protectorate concept quantitatively precise, provided that the material is in thermodynamic equilibrium and contains large numbers of interacting particles. In the broader space of out-ofequilibrium and nanoscale materials, we do not yet have such rigor, but there are many hints of protectorate-like behavior.

At this point, the reader is perhaps ready to turn from such philosophical considerations and ask, "OK, so what is complex adaptive materials research in practice?" What we have observed is that complex adaptive materials research is itself an emergent process, organically driven by the needs of the participating scientists. We have noticed that complex adaptive materials typically fall at the boundaries between the traditional disciplines, demanding "cross-pollinating" collaborative study by physicists, chemists, materials scientists, engineers, and biologists. While discovery is experimentally driven (as it always has been in science), theoretical and computational scientists steeped in reductionist tradition can still play extraordinarily useful roles in formulating precise descriptions of organizing principles.

\section{ICAM and the Articles in This Issue}

To facilitate interaction among the diverse disciplines that feed into this area, an organization has emerged: the Institute for Complex Adaptive Matter (ICAM, see http://icam.ucop.edu and Reference 17), whose form continues to evolve organically. The articles presented here either are derived from or have figured prominently in ICAM-sponsored workshops.

Our first article, by Schmalian and Wolynes, discusses the notion of "electronic mayonnaise," a general theoretical scenario for inducing intrinsic electronic heterogeneity, which was recently discovered experimentally in the cuprate superconductors as discussed in the second article, by Slezak et al. The terminology refers to the well-known microscopic basis for the microemulsion we call mayonnaise: oil and vinegar, which would prefer not to mix, are held together by amphiphiles (supplied by lecithin from egg yolk) that are hydrophobic on one side and hydrophilic on the other. "Electronic mayonnaise" describes the phenomenon of different electronic phases such as antiferromagnetism (in which magnetic moments alternate in direction from site to site) and charge doped states (which can be either metallic or have ordered insulating electronic density) coexisting via a self-generated amphiphile-like crossover region. Similar to mayonnaise, the antiferromagnetism ("oil") and the charge doped phase ("water") can coexist in highly complex morphologies. The key is having rather long-range interactions (found in the charged regions, for example, due to unscreened Coulomb coupling of charges), which serve to uniformly frustrate the ordering tendencies.

The idea that such disorder can be selfgenerated is truly remarkable, and time will tell whether the disorder of the superconducting gap discussed by Slezak et al. is an intrinsic property of the electron system or is driven by closely coupled atomic disorder. Schmalian and Wolynes also note several other analogies to microemulsions, and suggest, based upon their work from the quantum end, the possibility of a self-generated microemulsion glass, heretofore unseen. It appears that Schmalian and Wolynes may have possibly found the description of a new protectorate.

In our second article, Slezak et al. describe how they have used ultralowtemperature scanning tunneling microscopy (STM) to open a new window on the phenomenon of high-temperature superconductivity in particular and strongly interacting electronic materials more generally. By this we mean materials in which the average repulsive energy of electrons is comparable with or exceeds the average kinetic energy. These strongly interacting materials include the cuprate hightemperature superconductors, because their parent phase is an interaction-driven Mott insulator: effectively, the electrons are in a traffic jam because of high density and blocking of motion from a very strong local Coulomb repulsion.

This article reports on results from technology developed in the Davis laboratory: 1. The ability to examine simultaneously real space and Fourier-transformed STM conductances as a function of voltage. The former measures the local density of electronic states, and the latter provides information about the modulation of the density of states at suitable scattering vectors in momentum space. Essentially, at various wave vectors, which span the highest occupied Fermi surface in momentum space, standing waves of electronic density can be set up and sampled by the STM. These improved methods have also been used to sample checkerboard patterns of electron density with unit cells of periodicity $\sim 4$ times the underlying atomic unit cell.

2. Using STM to map out the real-space variation of not just electronic density, but also electronic gaps in the superconducting state. Perhaps the most spectacular discovery of the group was that for $\mathrm{Bi}_{2} \mathrm{Sr}_{2} \mathrm{CaCu}_{2} \mathrm{O}_{8+x}$ in a range of oxygen dopings $(x)$ between 0.1-0.19, there is intrinsic heterogeneity in the gap map at the nano-scale, a completely novel phenomenon.

This work has stimulated enormous experimental and theoretical effort, including a re-examination of what we know about other electronic materials. It provides perhaps the clearest example of why the study of the emergent properties of complex adaptive matter is (and must be) experimentally driven.

In our third article, three examples of recently discovered emergent phenomena in strongly interacting electron materials are discussed by Curro et al.; their identification of scaling behavior in these systems 
makes it possible to establish the magnetic origin of the emergent phenomena:

1. Emergent scaling of heavy electron itinerancy. Heavy electron metals are lanthanide/actinide intermetallics such as $\mathrm{Ce}_{x} \mathrm{La}_{1-x} \mathrm{CoIn}_{5}$, so called because they behave as though their mass is $100-1000$ times enhanced over free electrons. Although these electrons are almost localized, in terms of their enormous effective mass (infinite mass implies localization), they remain itinerant, as do the charge carriers in any simple metal like aluminum, free to roam through the entire sample. The open $f$ shells display local moment behavior at high temperature, evidenced by a Curie-Weiss-like magnetic susceptibility characteristic of immobile electrons, and itinerant behavior at low temperature, where the magnetic susceptibility looks like an enhanced metallic Pauli (constant) behavior. The dividing point in temperature between these regimes is called the Kondo temperature scale $\left(T_{\mathrm{K}}\right)$. This Kondo scale appears both for a concentrated lattice limit $(x=1)$ and for a dilute limit $(x$ tending to zero). Analysis of nuclear magnetic resonance (NMR) Knight shift data for heavy electron alloys with variable $x$ shows a "two-fluid description" to be appropriate. In this analysis, a distinct temperature scale $\left(T^{*}\right)$ emerges in the fully concentrated limit describing itinerant electrons and associated with intersite magnetic interactions. The second "fluid" is described as localized heavy electrons associated with the dilute-limit Kondo scale. 2. Layered heavy electron superconductors acting like high-temperature superconductors. One of the great surprises of heavy electron metals is that despite the nearlocalization of the electronic quasi-particles, as measured by their huge mass, these same electrons can pair and induce superconductivity at low temperatures. By measuring and analyzing the NMR spin lattice relaxation rate, they show a common feature of the cuprate hightemperature superconductors and a class of layered cerium and-quite unexpected!- plutonium heavy electron superconductors: the spin lattice relaxation rate, which probes the loss of nuclear polarization to the electrons, is a function of temperature $T / T_{c}$, where $T_{c}$ is the transition temperature to the superconducting state. The same function describes both classes of materials, and the scaling description works out to $3-4 T_{c}$. The analysis suggests (1) a common origin of attractive electron pairing via antiferromagnetic correlations, and (2) that the heavy fermion superconductor's pairs have an anisotropic energy gap ( $d$-wave character) like their high-temperature cousins.
3. Scaling analysis of pseudogap behavior in high-temperature superconductors. The cuprate superconductors can be doped by varying their oxygen content, with an optimal oxygen concentration yielding the highest $T_{\mathrm{c}}$. The magnetic susceptibility of these materials below the optimal doping shows "pseudogap" behavior, achieving a peak at a temperature $T^{*}$ (not to be confused with the heavy electron scale just described) and then decreasing rapidly with temperature. Curro et al. summarize experimental data from a variety of probes (NMR, inelastic neutron scattering, angle-resolved photoemission spectra) to argue that the pseudogap behavior in fact corresponds to parts of the Fermi surface of highest occupied electron states participating in a kind of "incoherent" pairing below $T^{*}$, with coherent superconducting pairing resulting for the rest of the Fermi surface states at $T_{c}$. This work demonstrates the quantitative power that welldefined organizing principles can have.

In the fourth article, Ramirez examines the organizing principles of geometric frustration in the context of both magnetic and structural ordering. As alluded to previously, frustration refers to the inability of a system to satisfy all energy-lowering interactions. For example, magnetic moments on triangular lattice elements are generally frustrated if they experience antiferromagnetic interactions such that not all magnetic bonds can be satisfied. Ramirez points out that crystalline and well-ordered yet frustrated magnetic systems are characterized exclusively by structures with triangular subunits. Experimentally, this frustration is characterized by a magnetic susceptibility that shows a large and negative Curie-Weiss temperature (associated with local coupling) at high temperatures, but a suppression or even absence of ordering down to very low temperatures.

Frustrated systems also possess considerable low-temperature entropy associated with an abundance of low-lying equivalent states. For example, assuming that anisotropy limits the magnetic moments to point up or down, a single frustrated antiferromagnetic triangle has a ground state which is sixfold degenerate (six states have the same energy). Indeed, as noted long ago by Pauling, ice presents a classically known nonmagnetic example in which frustration is associated with the ambivalence of proton positions in hydrogen bonds. Ramirez details the recent discovery of a magnetic analogue to ice, a system termed "spin ice." He then discusses how frustration can extend to displacement degrees of freedom, speculating that the negative-thermal-expansion ma- terial $\mathrm{ZrW}_{2} \mathrm{O}_{8}$ retains cubic symmetry to the lowest temperatures despite having underconstrained and nominally "floppy" structural units. Although at present the explicit source of frustration is a subject of ongoing study, the relevance of geometric frustration to this material seems very high.

The last article in this issue, by Cox et al., veers from studies of inanimate matter to living systems. It provides an overview of a body of results from materials research approaches to the study of protein aggregation phenomena seen in amyloid disorders such as Alzheimer's and mad cow disease. In these diseases, proteins misfold relative to their ordinary monomeric forms and aggregate into novel structures, some of which are "amyloid," or starch-like, so called because of their response to dyes that stain starch.

Postmortem studies have found in many cases large aggregates, or plaques, composed of one-dimensional "fibrillar" structures of proteins. These fibrils are, in a very real sense, protein nanotubes with a width on the order of $\sim 10 \mathrm{~nm}$.

Whether these aggregated structures or their precursors lead to toxicity is still under study, and there is no consensus of understanding on the origin of the pathology. Because aggregation of proteins is also observed in nonbiological environments, and because studies on living organisms suggest little biological control or regulation of amyloidogenic aggregation, an approach focusing on the physical and chemical aspects of these systems is clearly of interest and has yielded fruit. High-resolution electron microscopy and atomic force microscopy have provided evidence for protein oligomers which can be spherical, annular, or filamentary in morphology. The annular oligomers are of particular interest because to the extent that they can pierce cell membranes, they kill cells by acting as superfluous ion pores, admitting, for example, excess calcium into the cell. One suggestion is that the fundamental aggregating unit may be built from "left-handed $\beta$-helices," spiral structures of remarkably regular triangular cross section of $\sim 1.9 \mathrm{~nm}$ edge length, in which strands of peptide hydrogen bond to neighboring strands on the triangular edges. Although the authors primarily focus on the protein misfolding and aggregation discovered in diseased tissues, nature has found ways to usefully employ and control amyloid structures (e.g., spider silk), and researchers are also finding ways to exploit these materials in nanotechnology (for example, by growing extraordinarily regular nanowires inside amyloid fibrils). 


\section{Summary}

We hope that these articles inspire discussion of and increased participation in the study of complex adaptive matter, and that they make it clear that those of us who have joined such research are as much motivated by a sense of beauty in the discovery and observation as by potential practical outcomes.

We close by recalling a parable of $\mathrm{N}$. David Mermin, whose fictional Prof. Mozart despaired of what a global reductionist approach to matter had taught us, apart from providing a beautiful and detailed "archaeology" of the cosmos. ${ }^{18}$ Comparing the world around us to the collected plays of Shakespeare, he suggests that a global reductionist would first try to understand correlations between the 50,000 or so words found in the Bard's work. But then he or she would notice: there are far fewer (only 26!) fundamental letters making up those words. Certainly, understanding the letter-letter interactions would provide a detailed understanding of the plays! But wait: letters are made of lines, which are still smaller in number. Aha! On to the study of lines! But, oh my, lines are made up of points, which are there or not-what can be simpler than zero or one?

In contrast, the researcher of complex adaptive matter might first respond
"What beautiful plays!" and take it from there.

\section{Acknowledgments}

We thank Peter Wolynes for a most helpful critical reading and suggestions for improvement in an earlier version of this manuscript. We also acknowledge the support of ICAM and the U.S. National Science Foundation for an International Materials Institute Grant for the International Institute for Complex Adaptive Matter. D.L. Cox acknowledges the longterm support of the U.S. Department of Energy, Basic Energy Sciences, and the Division of Materials Research.

\section{References}

1. P.W. Anderson, Science 177 (1972) p. 393.

2. R.B. Laughlin, D. Pines, J. Schmalian, B.P. Stojkovic, and P. Wolynes, Proc. Nat. Acad. Sci. USA 97 (2000) p. 32.

3. R.B. Laughlin and D. Pines, Proc. Nat. Acad. Sci. USA 97 (2000) p. 28.

4. For an outstanding exposition of global reductionism, see S. Weinberg, Dreams of a Final Theory (Vintage Press, New York, 1992).

5. For an outstanding exposition of the argument that science is making a transition from the "golden age of reductionism" to the "age of emergence," see R.B. Laughlin, $A$ Different Universe (Basic Books, New York, 2005)

6. W.E. Caswell and G.P. LePage, Phys. Lett. 167B (1986) p. 437; J. Polchinski, “Effective Field
Theory and the Fermi Surface," http://arxiv.org e-print archive, hep-th/9210046 (1992; updated 1999; accessed March 2005).

7. S. Goedecker, Rev. Mod. Phys. 71 (1999) p. 1085. 8. For an overview of the CHARMM (Chemistry at HARvard Molecular Mechanics) program, see http:/ / www.charmm.org/document/ Charmm/Overview.Html (accessed March 2005.

9. For an overview of the AMBER (Assisted Model Building with Energy Refinement) program, see D.A. Pearlman, D.A. Case, J.W. Caldwell, W.R. Ross, T.E. Cheatham III, S. DeBolt, D.M. Ferguson, G. Seibel, and P.A. Kollman, Comp. Phys. Commun. 91 (1995) p. 1.

10. D.A.C. Beck and V. Daggett, Methods 34 (2004) p. 112.

11. V.S. Pande, I. Baker, J. Chapman, S.P. Elmer, S. Khaliq, S.M. Larson, Y.M. Rhee, M.R. Shirts, C.D. Snow, E.J. Sorin, and B. Zagrovic, Biopolymers 68 (2003) p. 91.

12. K.G. Wilson and J. Kogut, Phys. Rep. 12 (1974) p. 75; K.G. Wilson, Rev. Mod. Phys. 47 (1975) p. 773.

13. M. Haruta, N. Yamada, T. Kobayashi, and S. Iijima, J. Catal. 115 (1989) p. 301.

14. R. Moro, X.S. Xu, S.Y. Yin, and W.A. de Heer, Science 300 (2003) p. 1265.

15. J.M. McHale, A. Auroux, A.J. Perrotta, and A. Navrotsky, Science 277 (1997) p. 788.

16. R. Shankar, Rev. Mod. Phys. 66 (1994) p. 129.

17. G. Boebinger, D.L. Cox, A.J. Hurd, and D. Pines, MRS Bull. 29 (2004) p. 963.

18. N.D. Mermin, Phys. Today 43 (1990) p. 9.

\begin{abstract}
Daniel L. Cox, Guest Editor for this issue of MRS Bulletin, is a professor of physics at the University of California, Davis. During the 2004-2005 academic year, he was a visiting fellow at the Center for Theoretical Biological Physics at the University of California, San Diego. In his research career, he has carried out theoretical investigations of the heavy fermion and high-temperature superconductors, quantum impurity models, and problems at the interface between biology and physics, including electronic properties of DNA, metallo-organic molecules that mimic transition-metal centers in proteins, and protein aggregation phenomena, especially in the prion diseases.
\end{abstract}

He received a BS degree in physics from the University of Washington in 1979 and his PhD degree in theoretical physics from Cornell University in 1985. After a postdoctoral stint at UC-San Diego, he joined the physics faculty at Ohio State University in 1986 and the physics faculty at UC-Davis in 1997.

Cox has been the recipient of an Alfred P. Sloan Fellowship (1988), a National Science Foundation Presidential Young Investigator Award (1988), and a John Simon Guggenheim Memorial Fellowship (2004). He was also a Troisieme Cycle Lecturer at the University of Lausanne in 1996. He is the deputy director for international matters of the Institute for Com- plex Adaptive Matter, a multicampus research program of the University of California. He served on the American Physical Society Panel on Public Affairs (2001-2004) and as a member at large of the executive committee of the Division of Condensed Matter of the American Physical Society (1999-2001).

Cox can be reached by e-mail at cox@ physics.ucdavis.edu.

David Pines, Guest Editor for this issue of MRS Bulletin, is the founding co-director of the Institute for Complex Adaptive Matter (a multicampus research program of the University of California) as well as a research professor of physics at the University of Illinois at Urbana-Champaign

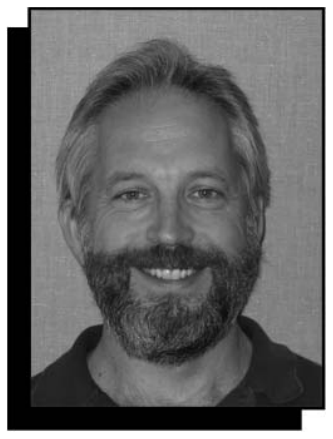

Daniel L. Cox

and a staff member at Los Alamos National Laboratory.

His contributions to the theory of many-body systems and theoretical astrophysics have been recognized by two John Simon Guggenheim Fellowships, the Eugene Feenberg Medal, and the Friemann, Dirac, and Drucker Prizes. Additionally, he has been elected to the National

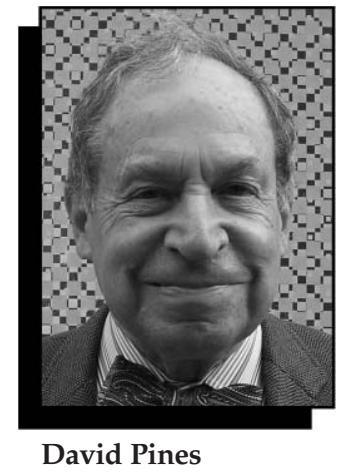

Academy of Sciences, the American Philosophical Society, the American Academy of Arts and Sciences, the Russian Academy of Sciences, and the Hungarian Academy of Sciences. He held visiting professorships at the Université de Paris, the University of Leiden, the College de France, Caltech, Trinity College, and 
the University of

Cambridge.

Pines can be reached

by e-mail at david.

pines@gmail.com.

Nicholas Curro is a technical staff member in the Condensed Matter and Thermal Physics group at Los Alamos National Laboratory. He has conducted NMR research for 12 years, studying several correlated electron materials including the hightemperature superconducting cuprates as well as heavy fermion intermetallic compounds.

He holds a BA degree from Rice University (1993), a master's degree in physics from the University of Illinois at Urbana-Champaign (1994), and a PhD degree in physics from UIUC (1998), where he did his thesis work in magnetic resonance with C.P. Slichter, investigating high-temperature superconductors in the normal and superconducting states.

Curro has more than 30 publications in refereed journals and has presented over 20 invited talks both in the United States and abroad.

Curro can be reached by e-mail at curro@ lanl.gov.

J.C. Davis is a professor in the Physics Department at Cornell University. He received his BSc degree from the National University of Ireland in 1983 and his PhD degree in physics from the University of California, Berkeley, in 1989 while working as a research assistant. He then worked as a postdoctoral research associate at Berkeley before accepting a position as an assistant professor of physics in 1993 . He became a faculty physicist at the Berkeley National Laboratory in conjunction with his promotion to associate professor, and he was later promoted to full professor. In 2003, he left Berkeley to take up his current position at Cornell.

Davis's research focuses on the application of experimental techniques of ultralow-temperature physics to questions of fundamental significance, which currently includes the study of atomic-scale phenomena in exotic superconductors using scanning tunneling microscopy, quantum nanofluidics and its applications in superfluids, two-dimensional superfluidity and superconductivity, and the ultimate sensitivity limits of mechanical systems to forces and accelerations. Among his honors are the NSF National Young Investigator Award (1994), the

Packard Fellowship in Science and Engineering (1994), the Alfred P. Sloan Research Fellowship (1994), the Miller Research Professor (2000), the Outstanding Performance Award from the Berkeley National Laboratory (2001), and the

Fritz London Memorial Prize (2005). He was an Ehrenfest Lecturer at the University of Leiden in Holland in 2002.

Davis can be reached by e-mail at jcdavis@ ccmr.cornell.edu.

Zachary Fisk is a professor of physics at the University of California, Davis. Following an AB degree from Harvard, he earned his PhD degree from the University of California, San Diego, with Bernd Matthias. $\mathrm{He}$ is a recipient of the E.O. Lawrence Award from the U.S. Department of Energy and the

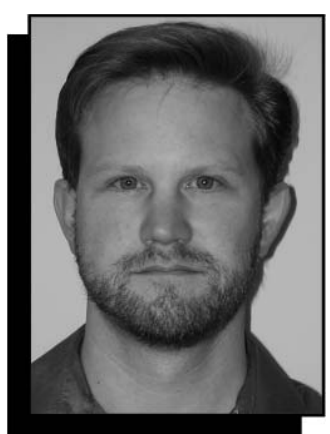

Nicholas Curro

American Physical Society New Materials Prize, and is a member of the National Academy of Science. He specializes in correlated electron physics, particularly the heavy fermion behavior of $4 f$ and $5 f$ intermetallics. Much of this research involves the search for new intermetallics and their characterization.

Fisk can be reached by e-mail at zfisk@ ucdavis.edu.

Hilal Lashuel is an assistant professor of neuroscience at the Integrative Biascience Institute, Ecole Polytechnique Fédérale de

Lausanne (EPFL) in

Lausanne, Switzerland, and director of the laboratory of molecular neurobiology and functional neuroproteomics. He received his BSc degree in chemistry from the City University of New York in 1994 and completed his doctoral studies at Texas A\&M University and the Scripps Research Institute in 1999. He was a postdoctoral research fellow at the Picower Institute for Medical Research in Long Island, N.Y., where he focused on developing amyloid-based therapeutic strategies for Alzheimer's disease.

In 2001, he moved to

Harvard Medical School and the Brigham and

Women's Hospital as a

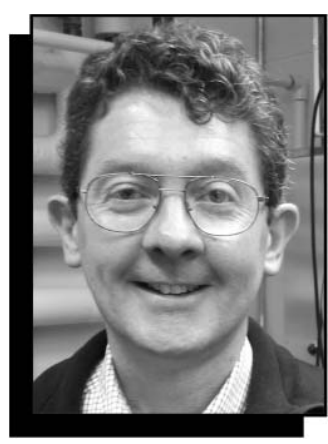

J.C. Davis

research fellow in the laboratory of Peter T. Lansbury at the Center for Neurologic Diseases. That same year, he received a sabbatical fellowship from the Harvard Center for Neurodegeneration and Repair and was later promoted to instructor in neurology at Harvard Medical School and the Brigham and Women's Hospital.

Lashuel's current research interests are elucidating the structural basis of amyloidassociated toxicity with the aim of developing mechanism-based therapeutic strategies for treating neurodegenerative diseases, including Alzheimer's, Parkinson's, and Huntington's disease; understanding the role of quaternary structure in protein function and disease; and exploiting amyloid fibril formation for constructing polypeptide materials with potential applications in biotechnology and medicine.

Lashuel can be reached by e-mail at hilal.lashuel@epfl.ch.

Jinho Lee has been working as a postdoctoral research associate in the Davis group at Cornell University since 2003. He received his BS and MS degrees in physics from Seoul National University in 1992 and

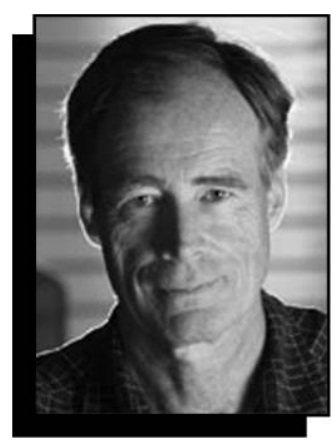

Zachary Fisk

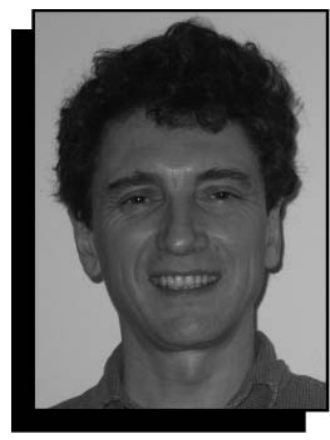

Arthur P. Ramirez

1994, respectively. After fulfilling his military service duty, he earned a $\mathrm{PhD}$ degree in physics from the University of Texas at Austin in 2002, where he built a lowtemperature STM system. He is currently working on low-temperature, extreme-precision scanning tunneling microscopy.

Lee can be reached by e-mail at j1453@ cornell.edu.

Ka Yee C. Lee is an associate professor in the Department of Chemistry, the Institute for Biophysical Dynamics, and the James Franck Institute at the University of Chicago. Her research focuses on the interaction of lipids with proteins or polymers at interfaces. She has carried out biophysical studies to elucidate the role of membranes in $\beta$-amyloid aggregation, the functioning of lung surfactants, the targeting selectivity 


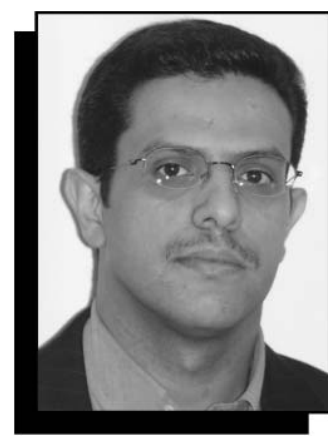

Hilal Lashuel

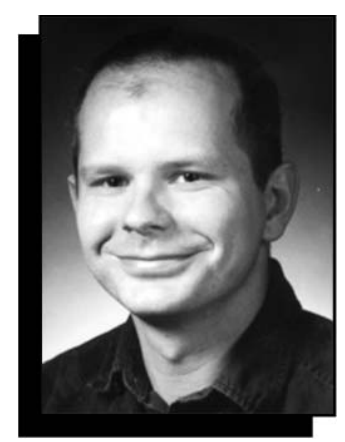

Joerg Schmalian

of antimicrobial peptides, the mechanism of membrane sealing by polymers, and the interaction of cholesterol with lipid membranes.

She received her ScB degree in electrical engineering from Brown University in 1986, and her MS and $\mathrm{PhD}$ degrees in applied physics from Harvard University in 1987 and 1992, respectively. Lee did her first postdoctoral training in chemistry at Stanford University and her second in chemical engineering at the University of California, Santa Barbara. In 1998, she joined the faculty of the University of Chicago.

Lee has been the recipient of a Dreyfus New Faculty Award (1998), a March of Dimes Basil O'Connor Starter Scholar Research Award (1999), a Searle Scholar Award (1999), a David and Lucile Packard Fellowship for

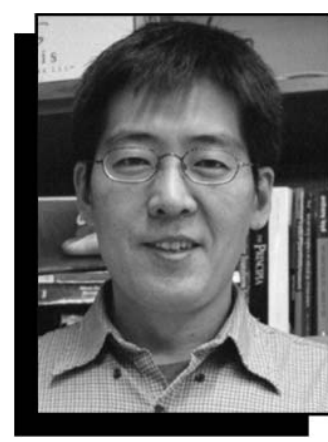

Jinho Lee

Science and Engineering (1999), the Ruth Salta Junior Investigator Achievement Award in Alzheimer's Disease Research from the American Health Assistance Foundation (1999), the Margaret Oakley Dayhoff Award from the Biophysical Society (2001), and an Alfred P. Sloan Fellowship (2001). She serves on the executive committee and the scientific steering committee of the Institute for Complex Adaptive Matter and as a member at large of the executive committee of the

Division of Biological Physics of the American Physical Society.

Lee can be reached by e-mail at kayeelee@ uchicago.edu.

Arthur P. Ramirez is director of the Condensed Matter Physics Research Department at Bell Laboratories, Lucent Technologies, as well as an adjunct professor at Columbia University. He earned his BS and $\mathrm{PhD}$ degrees in physics from Yale University, and except for a threeyear hiatus spent as a group leader at Los Alamos National Laboratory, he has spent his entire career at Bell.

His interests include crystalline organic semiconductors, fullerene superconductivity, thermoelectrics, multi-

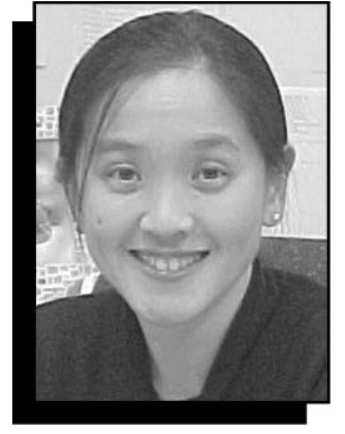

Ka Yee C. Lee

ferroics, colossal magnetoresistance, 2D electron gases, and heavy fermions.

Ramirez can be reached by e-mail at apr@lucent.com.

Joerg Schmalian is an associate physicist at Ames Laboratory in Ames, Iowa, and an associate professor in physics and astronomy at Iowa State University. He earned his undergraduate degree in physics from the University of Merseburg in Germany in 1990 and his PhD degree in physics from the Freie Universität Berlin in 1993. He was a visiting scientist at LEPES-CNRS in Grenoble, France, in 1995, followed by an appointment as a postdoctoral research associate and visiting research assistant professor at the University of Illinois at Urbana-Champaign in 1997. He was also a staff scientist at the Rutherford Appleton Laboratory in Oxfordshire, United Kingdom.

His research activities focus on theoretical condensed-matter physics, specifically in the investigation of strongly correlated, many-body systems and new collective behavior emerging due to competing interactions. $\mathrm{He}$ is working on phenomena such as magnetism, disordered systems, and nonequilibrium dynamics in complex materials including polymers, microemulsions, correlated electron systems, and glasses.

Schmalian has received numerous awards, including the Iowa State Foundation's Early Achievements in Research Award and the Daimler Benz Corporation's Carl Ramsauer Research Award. In addition, he is a member of the Institute for Complex Adaptive Matter's international advisory committee and the scientific steering committee, and he has organized or co-organized various workshops, including the Unconventional Superconductivity Workshop (2004) and the Workshop on Complexity in Strongly Correlated Electron Systems (2005). He has authored or co-authored articles for such journals as Physical Review Letters and Chemical Physics Letters.

Schmalian can be reached by e-mail at schmalian@ameslab.gov.

Rajiv R.P. Singh is a professor in the Department of Physics at the University of California, Davis. He received his BSc degree in physics from the Indian Institute of Technology in Kharagpur, India, his MSc degree in physics from Delhi University, and his $\mathrm{PhD}$ degree in physics, from the State University of New York at Stony Brook. While completing his $\mathrm{PhD}$ degree at SUNY-Stony Brook, he also held a position as a teaching/ research assistant. After receiving his $\mathrm{PhD}$ degree, he was a postdoctoral associate at Cornell
University and then the University of Maryland before becoming a postdoctoral member of the technical staff at Bell Laboratories. Singh accepted a position in 1989 as assistant professor in the Department of Physics at UC-Davis, where he was promoted to associate professor and then to his current position as full professor.

Singh has been honored as a Gordon Godfrey Visiting Professor and Visiting Fellow at the University of New South Wales in Sydney, Australia, and a visiting fellow at Princeton University. He has received the T.A. Pond Prize from SUNY-Stony Brook, the K.S. Krishnana Gold Medal from Delhi University, and the Institute Silver Medal from the IIT Kharagpur. In addition, Singh has authored or co-authored more than 100 publications, coordinated or co-organized multiple workshops and conferences, and mentored or co-supervised numerous PhD students and postdoctoral researchers. He also acts as a reviewer for various physics and chemistry journals.

Singh can be reached by e-mail at singh@ physics.ucdavis.edu.

James A. Slezak received his BS degree from the University of Sydney, Australia, in 2000, with first class honors in pure mathematics. He was awarded an MS degree in physics from Cornell University in 2005 and is a continuing PhD candidate. He has been researching hightemperature superconductivity by scanning tunneling microscopy in the Davis group at Cornell since 2003. 
Slezak can be reached by e-mail at james. slezak@cornell.edu.

Peter G. Wolynes is the Francis Crick Professor of the Physical Sciences chemistry/biochemistry at the University of California, San Diego. He received his $\mathrm{AB}$ degree in 1971 from Indiana University and his AM degree in 1972 from Harvard University, both in chemistry. He remained at Harvard until 1976, where he obtained his $\mathrm{PhD}$ degree in chemical physics. He then completed postdoctoral studies at the Massachusetts Institute of Technology before returning to Harvard as an assistant and a professor of professor. He was later promoted to associate professor. In 1980,

Wolynes accepted a position at the University of Illinois as an associate professor of chemistry and, in 1983, was promoted to full professor of chemistry, physics, and biophysics. He remained at the University of Illinois until he accepted his current position at UC-San Diego in 2000. honored as an Alfred P. Sloan Fellow, a John Simon Guggenheim Fellow, an honorary doctor of science at Indiana University, and a Fogarty Scholar in Residence by the National Institutes of Health. He is also a
Wolynes has been

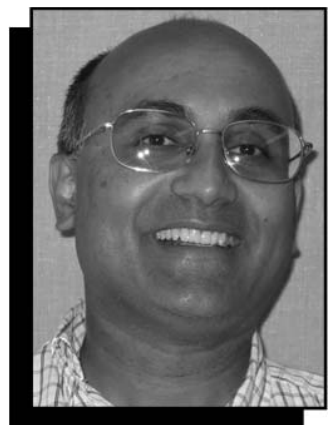

Rajiv R.P. Singh

member of the National Academy of Sciences, a fellow of the American Academy of Arts and Sciences, the American Association for the Advancement of Science, the Biophysical Society, and the American Physical Society. His awards include the American

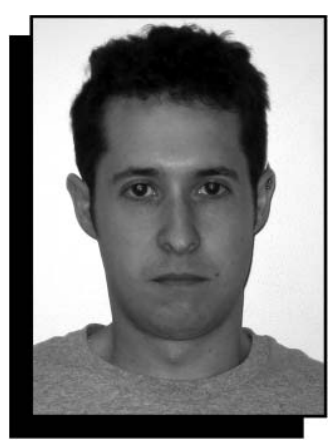

James A. Slezak

Chemical Society Award in Pure Chemistry, the Fresenius Award of Phi Lambda Upsilon, and the Peter Debye Award in Physical Chemistry of the American Chemical Society. In addition, he has authored or coauthored numerous proceedings and articles for

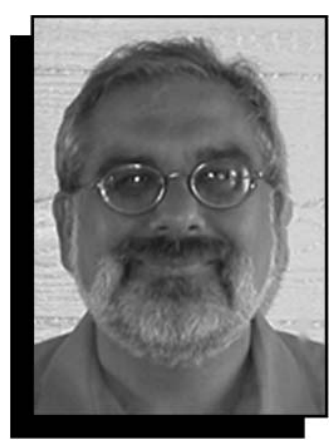

Peter G. Wolynes

such journals as Journal of Molecular Biology, Physics Review Letters, Journal of the American Chemical Society, and Europhysics Letters.

Wolynes can be reached by e-mail at pwolynes@ucsd.edu.

\section{MRS Materials Connections} www.mrs.org/connections/

Your source for Materials Research-Related Information! Research news, materials data sources, materials Web site database, meetings calendar, and more.
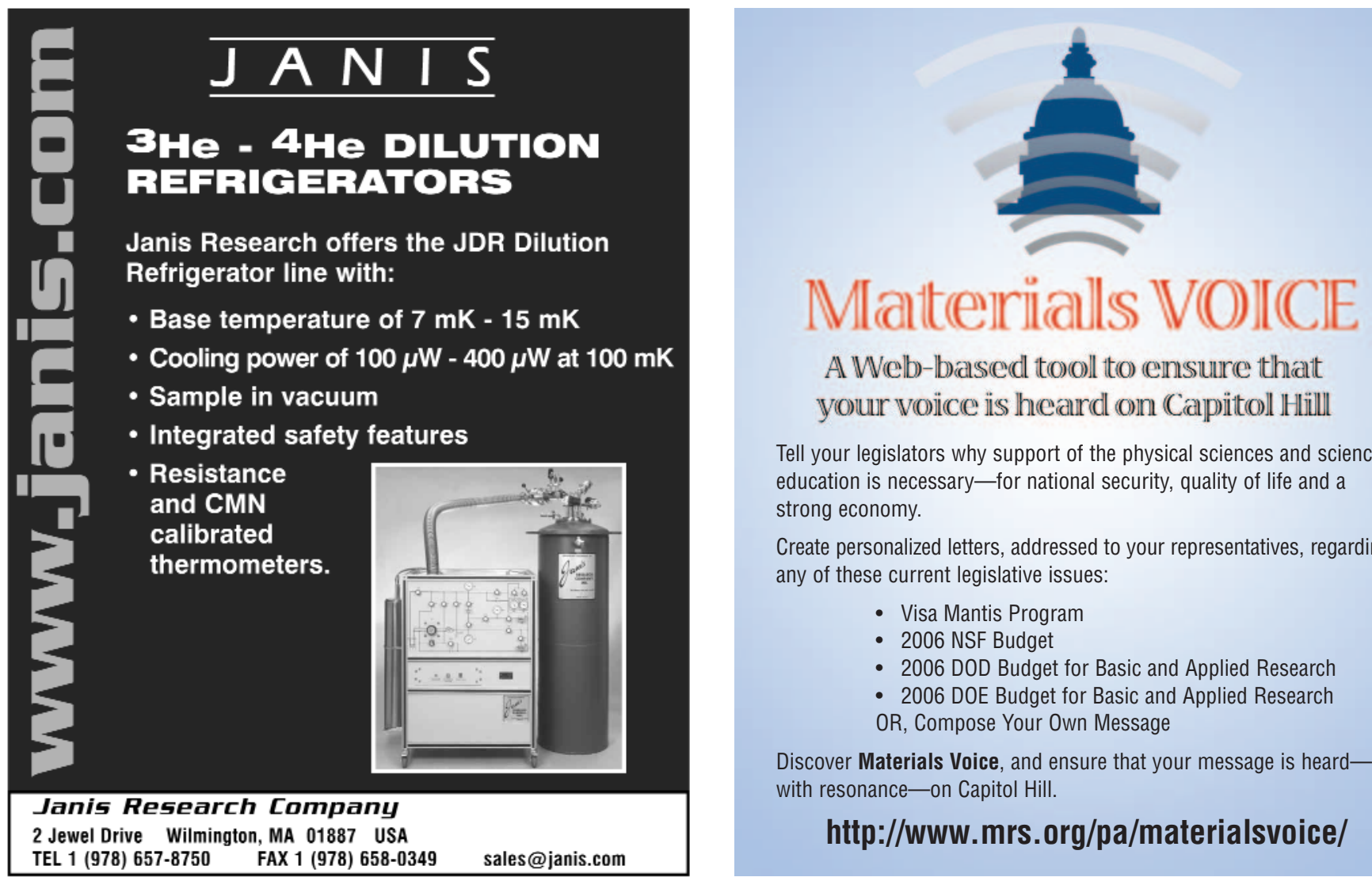

\section{Mlaitcerialls $\mathbb{V}(\mathrm{O}) \mathrm{I}(\mathrm{CIE}$ \\ A Web-brassed tooll tio ensurure that your voilice is heaurd on (Caupititoll Hillll}

Tell your legislators why support of the physical sciences and science education is necessary - for national security, quality of life and a strong economy.

Create personalized letters, addressed to your representatives, regarding any of these current legislative issues:

$$
\begin{aligned}
& \text { - Visa Mantis Program } \\
& \text { - } 2006 \text { NSF Budget } \\
& \text { - } 2006 \text { DOD Budget for Basic and Applied Research } \\
& \text { - } 2006 \text { DOE Budget for Basic and Applied Research } \\
& \text { OR, Compose Your Own Message }
\end{aligned}
$$

Discover Materials Voice, and ensure that your message is heardwith resonance- on Capitol Hill.

\section{http://www.mrs.org/pa/materialsvoice/}

For more information, see http://advertisers.mrs.org 